\title{
Rectification of Swimming Bacteria and Self Driven Particle Systems by Arrays of Asymmetric Barriers
}

\author{
M.B. Wan ${ }^{1,2}$, C.J. Olson Reichhardt ${ }^{1}$, Z. Nussinov ${ }^{1,2}$, and C. Reichhardt ${ }^{1}$ \\ ${ }^{1}$ Theoretical Division, Los Alamos National Laboratory, Los Alamos, New Mexico 87545 \\ ${ }^{2}$ Department of Physics, Washington University, St Louis, Missouri 63160
}

(Dated: November 2, 2018)

\begin{abstract}
We show that the recent experimental observation of the rectification of swimming bacteria in a system with an array of asymmetric barriers occurs due to the ballistic component of the bacteria trajectories introduced by the bacterial "motor." Each bacterium selects a random direction for motion and then moves in this direction for a fixed period of time before randomly changing its orientation and moving in a new direction. In the limit where the bacteria undergo only Brownian motion on the size scale of the barriers, rectification does not occur. We examine the effects of steric interactions between the bacteria and observe a clogging effect upon increasing the bacteria density.
\end{abstract}

PACS numbers: 87.17.Jj,05.40.Fb

Recent experiments have shown that swimming bacteria undergo a rectification effect when placed in a container that has an array of funnel-shaped barriers [1]. The initial bacteria density $\rho_{b}$ is constant throughout the container, but over time, bacteria accumulate on one side of the container due to interactions with the barriers. The ratio $r=\rho^{(1)} / \rho^{(2)}$ of the bacteria density $\rho^{(1)}$ on one side of the container to the density $\rho^{(2)}$ on the other side of the container increases over time in the presence of a single line of barriers, reaching a saturation value of around $r=2.75$. By taking advantage of this rectification effect, it has been experimentally demonstrated that various types of bacteria patterns can be induced to form, as well as closed circuits such as a bacteria pump created from a series of barrier arrays. If dead bacteria or genetically modified nonswimming bacteria are placed in the system instead of normal swimming bacteria, the rectification effect vanishes. The dead or nonswimming bacteria still undergo thermal motion and can be regarded as Brownian particles. In the experiments, a mixture of swimming and nonswimming bacteria was separated using the rectification effect of the barrier array.

The basic questions raised by these experiments are: (i.) what are the crucial properties necessary to give rise to rectification of the swimming bacteria and (ii.) what prevents the nonswimming bacteria from undergoing rectification. Possible factors that could be important include hydrodynamic interactions with the barrier walls, the details of the placement of the driving motor on the bacteria, the elongated shape of the bacteria, the distance that swimming bacteria move before tumbling and selecting a new swimming direction, and the dimensions of the asymmetric barriers. Additionally, other complications such as bacterium-bacterium interactions and chemical sensing could also play a role in the rectification. In Ref. 1], the rectification is attributed to a funnel mechanism arising from interactions between the bacteria and the barriers.

Motivated by ideas in Ref. [1], in this work we study a simple model for pointlike swimming bacteria which produces rectification in the presence of asymmetric barriers. Each bacterium moves ballistically under the influence of a motor force in a randomly chosen direction for a fixed distance $l_{b}$ before selecting a new random direction and moving ballistically again. This type of motion is known to occur for swimming bacteria which move in a fixed direction for a period of time before undergoing a tumbling locomotion and moving in a new direction 2]. We assume that upon interacting with a barrier, the bacterium does not reorient but moves along the barrier wall at a velocity determined by the component of the motor force that is parallel to the wall. As a result, the bacteria are entrained along the barrier walls as in Ref. [1] and rectification of the bacteria density occurs. The rectification persists even when an additional Langevin noise term is added to the ballistic motion of the bacteria.

For a system with a single line of barriers, we show that the magnitude of the rectification increases with increasing ballistic flight distance $l_{b}$, while in the limit where the bacteria move like Brownian particles on the length scale of the barriers, the rectification vanishes. We also find that in this model the bacteria density is highest along the container walls, in agreement with experimental observations. When steric interactions between the bacteria are included, the rectification is reduced due to a clogging effect. We show that the experimental system is a realization of a correlation ratchet [3], in which particles with fluctuations that violate detailed balance can give rise to a ratchet effect in the presence of an additional asymmetry such as an asymmetric substrate. Our model should also be useful for other systems of self-propelled particles where the particle motion is non-Brownian on a certain length scale. Recent examples of such systems include self-propelled colloids [4], artificial swimmers made from magnetic beads [5], autonomously moving catalytic nanorods [6], and self-propelled nematics [7]. Since the amount of rectification is affected by the ballistic flight length $l_{b}$, the asymmetric barriers could be used to sort 


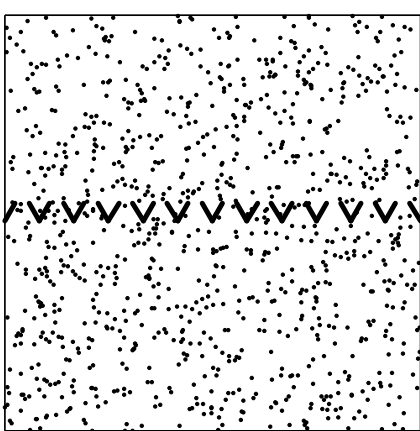

(a)

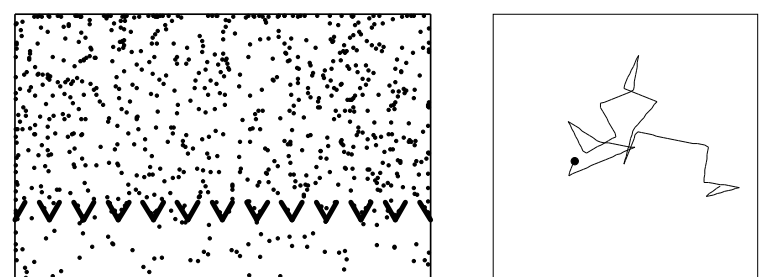

(a)

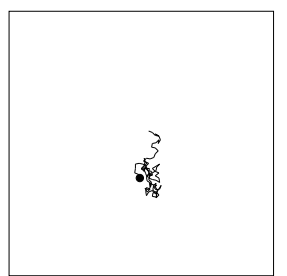

(b)

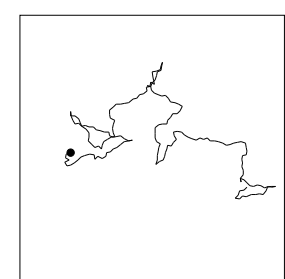

(c)
FIG. 2: Dots: position of one bacterium in the system. Lines: trajectory of the bacterium over a fixed time period. (a) A system with only a motor force showing ballistic motion and a random reorientation each time the bacterium has moved a distance $l_{b}=10$. Here $F^{T}=0$. (b) A system with small $l_{b}=1$ and $F^{T}=0$. (c) A system with both ballistic motion and thermal motion, where $l_{b}=10$ and $F^{T}=10$. dots: bacteria positions; heavy black lines: barrier locations. (a) Initial system configuration with uniform bacteria density for $N_{b}=980$ and $l_{b}=40$. The density in the upper chamber is denoted $\rho^{(1)}$ and the density in the lower chamber is $\rho^{(2)}$. (b) The same system after $100 \tau$ simulation steps showing the higher bacteria density in the upper chamber, $\rho^{(1)}>\rho^{(2)}$.

different species of swimming or self-propelled particles.

Our simulation consists of a bounded two-dimensional box of an array of funnel shaped barriers as shown in Fig. 1. The system has dimensions of $L \times L$, where $L=99$ unless otherwise noted, and contains $N_{b}$ particles representing bacteria at an overall density $\rho_{b}=N_{b} / L^{2}$. The particles move in an overdamped media where we do not take into account any hydrodynamic effects. A single bacterium $i$ obeys the following equation of motion:

$$
\eta \frac{d \mathbf{R}_{i}}{d t}=\mathbf{F}_{i}^{m}(t)+\mathbf{F}_{i}^{T}+\mathbf{F}_{i}^{B}+\mathbf{F}_{i}^{S}
$$

Here $\mathbf{R}_{i}$ is the position of bacterium $i$ and $\eta$ is the phenomenological damping constant which we set to $\eta=1$. The driving force from the bacterial motor $\mathbf{F}_{i}^{m}(t)$ has a constant magnitude $\left|\mathbf{F}^{m}\right|=2.0$ but is applied in a randomly chosen direction which changes after every $\tau$ simulation time steps. In the absence of other forces the bacterium would move ballistically over a distance $l_{b}=\tau \delta t\left|\mathbf{F}^{m}\right|$ before reorienting, where $\delta t=0.005$ is the magnitude of a simulation time step. The resulting random walk motion is illustrated in Fig. 2(a) for $\tau=1000$ and $l_{b}=10$. As $\tau \rightarrow 1$, the motion approaches a random walk even at the smallest size scales, as shown in Fig. 2(b) for $l_{b}=1$. The thermal force arising from fluctuations in the solvent is given by $\mathbf{F}_{i}^{T}$, which has the properties $\left\langle F_{i}^{T}(t)\right\rangle=0$ and $\left\langle F_{i}^{T}(t) F_{j}^{T}\left(t^{\prime}\right)\right\rangle=2 \eta k_{B} T \delta_{i j} \delta\left(t-t^{\prime}\right)$. The bacteria motion under the influence of both thermal fluctuations and the ballistic motor force is illustrated in Fig. 2(c) for $l_{b}=10$ and $F^{T}=10$. For most of this work we set $F^{T}=0$. The force from the barriers and container walls is given by $\mathbf{F}_{i}^{B}$. Each barrier is modeled by two half-parabolic domes of strength $f_{B}=30$ and radius $r_{B}=0.05$ separated by an elongated region of length $L_{B}=5.0$ which repels the bacteria perpendicular to the trap axis: $\mathbf{F}_{i}^{B}=\sum_{k=0}^{N_{B}}\left[\frac{f_{B} r_{1}}{r_{B}} \Theta\left(r_{1}\right) \hat{\mathbf{R}}_{i k}^{ \pm}+\frac{f_{B} r_{2}}{r_{B}} \Theta\left(r_{2}\right) \hat{\mathbf{R}}_{i k}^{\perp}\right]$.
Here the total number of barriers (including confining walls) is $N_{B}=28, r_{1}=r_{B}-R_{i k}^{ \pm}, r_{2}=r_{B}-R_{i k}^{\perp}$, $R_{i k}^{ \pm}=\left|\mathbf{R}_{i}-\mathbf{R}_{k}^{B} \pm L_{B} \hat{\mathbf{p}}_{\|}^{k}\right|, \hat{\mathbf{R}}_{i k}^{ \pm}=\left(\mathbf{R}_{i}-\mathbf{R}_{k}^{B} \pm L_{B} \hat{\mathbf{p}}_{\|}^{k}\right) / R_{i k}^{ \pm}$, $\mathbf{R}_{i}\left(\mathbf{R}_{k}^{B}\right)$ is the position of bacterium $i$ (barrier $k$ ), and $\hat{\mathbf{p}}_{\|}^{k}\left(\hat{\mathbf{p}}_{\perp}^{k}\right)$ is a unit vector parallel (perpendicular) to the axis of barrier $k$. Each funnel is modeled as two barriers meeting at a common endpoint and placed at angles $\theta$ and $\pi-\theta$ with the $x$-axis. Our system contains 12 funnels in a one-dimensional array with a lattice constant of $l_{S}=8.25$. The steric force from the bacteriumbacterium interaction, $\mathbf{F}_{i}^{S}$, is modeled as a stiff repulsive spring of range $r_{s}=0.35$ and strength $f_{s}=150$, with $\mathbf{F}_{i}^{S}=\sum_{j \neq i}^{N_{b}} \frac{f_{s} r_{3}}{r_{s}} \Theta\left(r_{3}\right) \hat{\mathbf{R}}_{i j}$. Here $r_{3}=r_{s}-R_{i j}$, $R_{i j}=\left|\mathbf{R}_{i}-\mathbf{R}_{j}\right|$, and $\hat{\mathbf{R}}_{i j}=\left(\mathbf{R}_{i}-\mathbf{R}_{j}\right) / R_{i j}$. For most of this work we consider the limit where bacteriumbacterium interactions are irrelevant and set $f_{s}=0$.

We base our system geometry on the work in Ref. [1]. In Fig. 1(a) we illustrate the system geometry, showing the array of funnel shapes, the boundary walls, and the initial locations of the bacteria. The bacteria density in the upper (lower) chamber is $\rho^{(1)}\left(\rho^{(2)}\right)$. The angle $\theta$ of each funnel barrier is set to $\theta=60^{\circ}$. We first investigate the simplest set of parameters that produce rectification by fixing $F^{T}=0, f_{s}=0$, and $l_{b}=40$. The initial density ratio $r=\rho^{(1)} / \rho^{(2)}=1$, as shown in Fig. 1(a). In Fig. 1(b) we illustrate the same system after $100 \tau$ time steps when $r=2.3$, showing a density increase in the upper half of the sample. A buildup of bacteria occurs along the barrier walls and the boundaries, similar to that observed in the experiments of Ref. [1].

In Fig. 3(a) we plot the time dependent behavior of $\rho^{(1)} / \rho_{b}$ and $\rho^{(2)} / \rho_{b}$ for the same system shown in Fig. 1 with $l_{b}=180$. We also show the same quantities for a system with a much smaller $l_{b}=0.01$ in which the bacteria motion is equivalent to a random walk on the size scale of the barriers. For the small $l_{b}$ there is no rectification of the bacteria density as would be expected, while $R_{i k}^{\perp}=\left|\left(\mathbf{R}_{i}-\mathbf{R}_{k}^{B}\right) \cdot \hat{\mathbf{p}}_{\perp}^{k}\right|, \hat{\mathbf{R}}_{i k}^{\perp}=\left(\left(\mathbf{R}_{i}-\mathbf{R}_{k}^{B}\right) \cdot \hat{\mathbf{p}}_{\perp}^{k}\right) / R_{i k}^{\perp}$, 


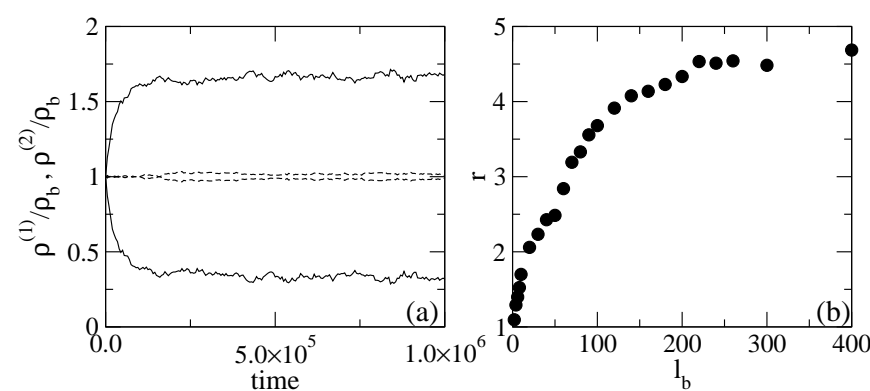

FIG. 3: (a) The time dependence of the bacteria density $\rho^{(1)} / \rho_{b}$ (upper line) and $\rho^{(2)} / \rho_{b}$ (lower line), normalized by the overall bacteria density $\rho_{b}$, for the same system in Fig. 1 with $l_{b}=180$, showing the density buildup over time in the top half of the sample. Dotted lines: $\rho^{(1)} / \rho_{b}$ and $\rho^{(2)} / \rho_{b}$ for the same system but with $l_{b}=0.01$ so that the bacteria undergo Brownian motion on the size scale of the barrier and no rectification occurs. (b) $r=\rho^{(1)} / \rho^{(2)}$ versus $l_{b}$ for a sample with $\theta=60^{\circ}$ measured after $10^{3} \tau$ simulation steps.

for the large $l_{b}$ the system rectifies. The time dependence of $r=\rho^{(1)} / \rho^{(2)}$ at short times can be fit to an exponential form, similar to the experiments of Ref. [1]. We note that asymptotically, $r \approx 4.3$, while the asymptotic value of $r$ depends on $l_{b}$. We have repeated the simulations for different values of $l_{b}$, and in Fig. 3(b) we plot $r$ versus $l_{b}$ measured after $10^{3} \tau$ simulation time steps. For low $l_{b}<200$, the rectification increases monotonically with $l_{b}$, with $r$ reaching a maximum of value of $r=4.8$ at $l_{b}=200$. We note that $r$ depends in a similar way upon the barrier length $L_{B}$ for fixed $l_{b}$, and the rectification vanishes for large values of $L_{B}$ when there is no longer a gap between adjacent funnel barriers.

These results suggest that hydrodynamic effects or chemotaxis are not required to produce the rectification seen in Ref. [1], but that instead the rectification arises from the fact that the bacteria move in finite steplike motions. In Fig. 4(a) we illustrate the specific mechanics that give rise to the funneling behavior of the bacteria at the barrier. As described in Ref. [1], a bacterium which collides with a barrier cannot continue to move in the direction of the motor force; instead, the bacterium slides along the barrier at a velocity determined by the component of the motor force parallel to the barrier. If $l_{b}$ is large enough, there is a high probability that the bacterium will slide all the way across the barrier to the other half of the container before the motor force changes its orientation. If $l_{b}$ is very small, the bacterium cannot move very far along the barrier before it randomly moves off when the motor force changes, as illustrated by the light line in Fig. 4(a). The rectification is produced by the combination of the asymmetric barrier and the motion of the bacteria that allows a breaking of detailed balance when the bacteria interact with the barriers. Another consequence of this model is that the bacteria should tend to accumulate on the container barrier walls, as observed in
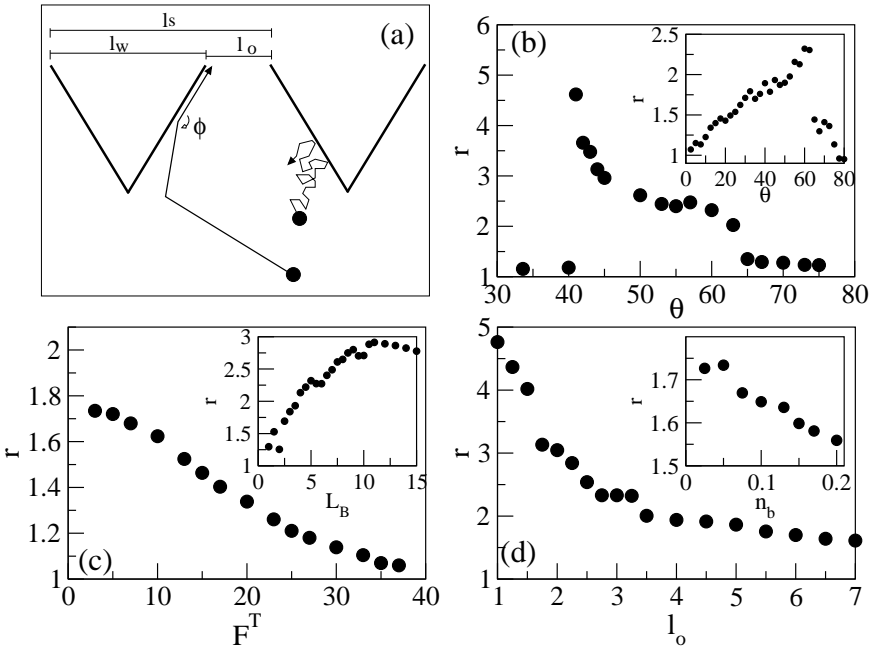

FIG. 4: (a) Schematic trajectories for: (dark line) a bacterium moving under motor forces only and being entrained by the wall; (light line) a bacterium experiencing only Brownian forces. The barrier spacing $l_{S}$, opening size $l_{o}$, funnel width $l_{w}$, and angle $\phi$ between the bacterium trajectory and the funnel barrier wall are also indicated. (b) $r=\rho^{(1)} / \rho^{(2)}$ vs $\theta$ for a system with $l_{b}=20, F^{T}=0, f_{s}=0$, and $L=99$. Inset: $r$ vs $\theta$ for the same system with fixed $l_{o}=3.25$ and varied $L$. (c) $r$ vs $F^{T}$ for a system with $l_{b}=20, \theta=60^{\circ}$, $L=99$, and $f_{s}=0$. Inset: $r$ vs $L_{B}$ for a system with $l_{b}=20$, $F^{T}=0, f_{s}=0$, fixed $l_{o}=3.25$, and varied $L$. (d) $r$ vs $l_{o}$ for a system with $l_{b}=20, \theta=60^{\circ}, L_{B}=5.0, f_{s}=0$, and varied $L$. Inset: $r$ vs overall bacteria density $\rho_{b}$ for a system with steric interactions, $f_{s}=150, \theta=60^{\circ}, f^{T}=0, L=99$, and $l_{b}=20$.

the experiment [1]. We note that the real bacteria may tend to be hydrodynamically trapped by the wall. This effect would tend to enhance the rectification effect since the bacteria could move for distances longer than $l_{b}$ along the barrier, generating rectification even for moderately small $l_{b}$.

To compare our simulation results quantitatively to experiment, we take a simulation distance unit of 1 micron and time step unit of $5 \times 10^{-4} \mathrm{sec}$. This gives a bacteria velocity of 20 microns/sec, close to the estimated speed $v \approx 23$ microns/sec for $E$. coli [8, 9]. A tumbling rate of roughly $1 \mathrm{~Hz}$ [8] corresponds to $l_{b}=20$ microns; Ref. [1] gives the estimate $l_{b} \approx 50$ microns. Fig. 3(a) shows that our system reaches a stationary state within $1 \times 10^{5}$ time steps, which corresponds to 50 minutes. This compares well with the saturation time scale in the experiment of 20 to 80 minutes [1].

We next consider changing the geometry of the barrier funnels. In Fig. 4(b) we plot $r$ vs $\theta$, the funnel angle, after $10^{3}$ simulation steps for a system with $l_{b}=20$ and fixed $L=99$. For $\theta<41^{\circ}$ adjacent funnel barriers are in contact so there is no transport. The maximum of $r=4.6$ occurs at $\theta=41^{\circ}$, and $r$ decreases with increasing $\theta$ for $\theta>41^{\circ}$, reaching $r=1$ at $\theta=90^{\circ}$. The experiments also 
showed no rectification for flat barriers [1]. The inset of Fig. 4(b) shows the dependence of $r$ on $\theta$ when the space between funnel barriers $l_{o}$ is held fixed, indicating that the maximum rectification occurs for $\theta=60^{\circ}$. The inset of Fig. 4(c) illustrates that $r$ initially increases with barrier length $L_{B}$ before saturating, while Fig. 4(d) shows that $r$ decreases as $l_{o}$ increases.

A simple heuristic expression gives a lower bound for the value of $r$ in the case when the spacing $l_{S}$ between adjacent funnel barriers is much smaller than the ballistic flight length, $l_{S} \ll l_{b}$. Assuming that the bacteria are entrained by any barrier wall which they strike, detailed balance implies that in the steady state at long times,

$$
r=\rho^{(1)} / \rho^{(2)}=\operatorname{Prob} .(\phi>\pi / 2)\left(l_{w} / l_{o}\right)+1 .
$$

Here, the tip-to-tip width of a single funnel barrier is $l_{w}=$ $l_{S}-l_{o}$, as indicated in Fig. 4(a). In Eq.(2), Prob. $(\phi>$ $\pi / 2)$ is the probability that whenever the bacteria hit the funnel barrier walls, they do so at a large enough angle $\phi$ such that they slide along the wall in an upward direction to emerge in the top portion of the sample, as shown in Fig. 4(a). Equation (2) leads to a value of $r$ which is strictly bounded from below by one, reflecting the rectification effect. By measuring Prob. $(\phi>\pi / 2)$ we have verified that Eq.(2) is indeed generally satisfied.

In the experimental system, the bacteria experience some additional Brownian fluctuations due to the solvent. We model this effect by setting $F^{T}>0$, producing the type of motion shown in Fig. 1(c). If only the thermal noise term is applied and the motor force $F^{M}=0$, we find no rectification. In Fig. 4(c) we show $r$ vs $F^{T}$ for a system with $l_{b}=20$ and barrier radius $r_{b}=0.5$. As $F^{T}$ increases, the rectification is slowly reduced, while for large enough $F^{T}$ the rectification is washed out and $r=1$. The destruction of the rectification occurs because the thermal term causes some of the bacteria to move away from the barriers rather than channeling along them, so the progress along the walls is reduced from the mechanism shown in Fig. 4(a).

The bacteria in the experimental system have a steric interaction with each other which prevents overlap. To mimic this effect we add a steric force interaction by setting $f_{s}>0$. In Fig. $4(\mathrm{~d})$ we plot $r$ vs bacteria density $\rho_{b}=N_{b} / L^{2}$ for the same system in Fig. 2 but with a finite steric interaction strength of $f_{s}=150$. As the density $\rho_{b}$ increases, the effective rectification is reduced due to a clogging effect since only a small number of bacteria can fit along the barrier wall. The effect of the clogging can be reduced by the addition of thermal noise since this has a tendency to prevent bacteria from clustering.

We have also performed simulations for mixtures of interacting bacteria in which one species moves under both a motor force and thermal noise while the other species moves only due to thermal noise. This mimics a system with a combination of swimming and nonswimming bacteria. If the density of the nonswimming bacteria is sufficiently high, the rectification efficiency is reduced. It is also possible for an entrainment effect to occur in which the swimming bacteria can induce a weak rectification of the nonswimming bacteria.

The rectified motion in this system has similarities to Brownian ratchets where particles undergoing Brownian motion in the presence of an asymmetric potential can exhibit a net drift in the presence of an additional ac drive or flashing potential substrate [10]. Our system is not a Brownian ratchet in this sense; instead, it can be considered to be a realization of a correlation ratchet. In correlation ratchets, an overdamped particle can exhibit dc drift on an asymmetric substrate in the absence of an ac flashing or rocking provided that the fluctuations of the particle motion have certain properties that break detailed balance [3]. In bacteria and other self-propelled particle systems, it is the motor force that causes the fluctuations to break detailed balance.

To summarize, we show that a rectification phenomenon similar to that observed in recent experiments 1] can be achieved in a simple model for overdamped swimming bacteria where the bacteria move in straight lines of length $l_{b}$ before randomly changing direction while also interacting with funnel shaped barriers. In this model, no hydrodynamic interactions are needed to produce the rectification. In the limit of bacteria which undergo only Brownian motion on the size scale of the barriers, no rectification occurs, in agreement with experiment [1]. The rectification results when $l_{b}$ is large enough that bacteria which strike a funnel barrier move along the entire length of the barrier before the bacterial motor force changes direction. We have also examined the effects of steric interactions on the rectification and find a clogging phenomenon at the barrier funnels which reduces the effectiveness of the rectification. Additionally, if a sufficiently strong Langevin noise term is added to the model, the rectification is reduced. This system can be regarded as a two-dimensional realization of a correlation ratchet in which fluctuations that break detailed balance can give rise to directed motion in the presence of an asymmetric substrate. Our results should also be relevant to other two-dimensional systems of self-propelled particles that undergo a similar type of motion.

This work was carried out under the auspices of the NNSA of the U.S. DoE at LANL under Contract No. DE-AC52-06NA25396.

[1] P. Galajda, J. Keymer, P. Chaikin, and R. Austin, J. Bacteriol. 189, 8704 (2007).

[2] H.C. Berg, Random Walks in Biology (Princeton University Press, Princeton, NJ, 1983).

[3] C.R. Doering, W. Horsthemke, and J. Riordan, Phys. Rev. Lett. 72, 2984 (1994); M.M. Millonas and M.I. Dykman, Phys. Lett. A 185, 65 (1994). 
[4] J.R. Howse et al., Phys. Rev. Lett. 99, 048102 (2007).

[5] R. Dreyfus et al., Nature (London) 437, 862 (2005).

[6] P. Dhar et al., Nano Lett. 6, 66 (2006).

[7] S. Thakur et al., Phys. Rev. Lett. 97, 115701 (2006).
[8] N. Mittal et al., Proc. Natl. Acad. Sci. 100, 13259 (2003).

[9] N.C. Darnton et al., J. Bacteriol. 189, 1756 (2007).

[10] P. Reimann, Phys. Rep. 361, 57 (2002). 